\title{
Caracterização do perfil motor de escolares com transtorno autístico
}

\author{
Paola Matiko Okuda* \\ Andrea Regina Nunes Misquiatti** \\ Simone Aparecida Capellini ${ }^{\star \star *}$
}

\section{Resumo}

Foco temático: As alterações motoras podem fazer parte das chamadas comorbidades que podem coexistir com o transtorno autístico. Objetivo: caracterizar o perfil motor de escolares com espectro autístico. Método: participaram deste estudo 6 escolares com transtorno autístico do ensino fundamental, do gênero masculino, com idades variando entre 5 anos e 5 meses e 10 anos e 9 meses. Após a assinatura do termo de consentimento pelos pais ou responsáveis, os escolares foram submetidos à aplicação da Escala de Desenvolvimento Motor para avaliação da motricidade fina, motricidade global, equilíbrio, esquema corporal, organização espacial, organização temporal e lateralidade. Resultados: Os resultados revelaram diferença significante entre a idade motora e a idade cronológica. Conforme a classificação da Escala do Desenvolvimento Motor, os escolares deste estudo apresentaram desenvolvimento motor inferior ao esperado para a idade. Conclusão: Os escolares com transtorno autístico deste estudo apresentam quadro de Transtorno do Desenvolvimento da Coordenação em comorbidade, demonstrando que os participantes desta pesquisa apresentam dificuldades em atividades que exijam destreza como a grafia. Assim, as necessidades motoras e psicomotoras desses escolares são enfocadas no ambiente clínico e educacional para diminuir o impacto das manifestações comportamentais e sociais.

Palavras-chave: Transtorno da Coordenação Motora; Transtorno Autístico; Desenvolvimento Motor.

\section{Caracterization of the motor profile of students with autistic disorder}

\section{Abstract}

Thematic focus: The motor abnormalities may be part of so-called comorbidities that can coexist with autistic disorder. Objective: To characterize the motor profile of students with autistic disorder. Method: the study included six children with

\footnotetext{
* Terapeuta ocupacional. Aluna especial do Programa de pós-graducação em Educação (Mestrado) da Faculdade de Filosofia e Ciências da Univesidade Estadual Paulista. Marília, São Paulo, Brasil.

** Professora Doutora do Departamento de Fonoaudiologia e do Programa de pós-graducação em Educação (Mestrado) da Faculdade de Filosofia e Ciências da Univesidade Estadual Paulista. Marília, São Paulo, Brasil.

*** Professora Pós-Doudora do Departamento do Programa de pós-graducação em Educação (Mestrado) da Faculdade de Filosofia e Ciências da Univesidade Estadual Paulista. Marília, São Paulo, Brasil.
} 
autistic disorder in elementary school, male, aged 5 years and 5 months and 10 years and 9 months. After signing the consent form by parents or guardians, the students were submitted to the Motor Development Scale for assessment of fine motor, gross motor performance, balance, body scheme, spatial organization, temporal organization and laterality. Results: The results revealed a significant difference between the motor age and chronological age. According to the classification of the Scale of Motor Development, students in this study showed motor development lower than expected for age. Conclusion: The students with autistic disorder in this study presented a profile of Developmental Coordination Disorder in comorbidity, showing that participants of this research presented difficulties in activities that required skills such as handwriting. Thus, motor and psychomotor needs of these students were focused on educational and clinical environment to reduce the impact of behavioral and social manifestations.

Keywords: Developmental Coordination Disorder; Autistic disorder; Motor skills.

\section{Introdução}

De acordo com o Manual de Diagnóstico e Estatística das Perturbações Mentais (DSM-IV, 2002) e a Classificação Internacional das Doenças, $10^{\mathrm{a}}$ edição (CID-10), o termo transtorno autístico está inserido dentro dos transtornos invasivos do desenvolvimento (TID), que compreendem um amplo espectro de transtornos do desenvolvimento caracterizados pela presença de distúrbios do comportamento do início da vida com diferentes graus de gravidade e de déficits associados, que têm em comum a diminuição ou perda das habilidades sociais, da comunicação, da imaginação e do comportamento e a presença de interesses repetitivos e restritos.

As alterações motoras podem fazer parte das chamadas comorbidades que podem coexistir com o transtorno autístico (LARSON et al., 2008). O Transtorno do Desenvolvimento da Coordenação (TDC) é geralmente encontrado em crianças entre 6 e 12 anos de idade. Há cerca de dez anos, pesquisas estimaram que o TDC afetava de $10 \%$ a $19 \%$ de crianças em idade escolar; porém, atualmente, essa estimativa varia de $5 \%$ a $8 \%$ de crianças em idade escolar, tendo uma maior prevalência em meninos do que em meninas, na proporção de 2 para 1 (BARNHART et al., 2003; GABBARD; CAÇOLA, 2010).

Os sinais do TDC são geralmente associados a desajeitamento e à inconsistência no desempenho de tarefas, coordenação motora pobre, problemas de ritmo e sua transferência de aprendizagem, declínio do desempenho com a repetição, tensão corporal e excesso de atividade muscular em tarefas motoras, podendo apresentar dificuldades nas tarefas de auto-cuidado, como vestir-se, nas atividades acadêmicas, de lazer e esportes, além de problemas com interações sociais (MAGALHÃES; NASCIMENTO; REZENDE, 2004). 
Em crianças com transtorno autístico, o TDC pode ocorrer em decorrência de alterações nos padrões de sulcamento cotical nos lobos frontais e temporais, como também devido ao mau funcionamento em estruturas como o cerebelo, a amígdala, o hipocampo, no corpo caloso e no giro do cíngulo (LEVIT et al., 2003; ZILBOVÍCIUS; MERESSE, I.; BODDAERT, 2006; MELO et al. 2010). Tais regiões cerebais participam do processamento da informação referente ao ato motor (planejamento, sequenciação e execução) (KOLB; WHISHAW, 2002). Conforme descrito na literatura (MAGALLÓN; NARBONA, 2009), quando há funcionamento alterado do cerebelo, as habilidades visuo-espaciaias encontram-se comprometidas afetando diretamente as funções inerentes à aprendizagem da coordenação motora.

A capacidade motora é influenciada pela maturação nervosa que vai progressivamente determinando, de acordo com as características individuais, a capacidade de exercer suas funções, ou seja, a capacidade de representar ações mentalmente. Nos escolares com transtorno autístico, essa função está alterada e dessa forma o planejamento motor é deficitário (LIMA et al., 2001; GABBARD; CAÇOLA, 2010).

Segundo LARSON et al. (2008), as crianças com espectro autístico apresentam, entre outras características, alteração de coordenação motora, ocasionando atraso na aprendizagem de habilidades motoras finas e complexas (coordenação motora global, equilíbrio, esquema corporal, organização espacial e temporal).

Escolares com transtorno autístico mostram grande irregularidade na idade em que desenvolvem as sequências motoras ou de linguagem, o ritmo mais comum é uma descontinuidade na sequência normal do desenvolvimento. Também apresentam distúrbios da percep ᄀção e são incapazes de usar estímulos sensoriais para discriminar o que é importante ou não. Em outras palavras, ocorre um erro de seletivi-dade, prejudicando, assim, a função motora eficiente (FERNANDES, 2008).

Com base no exposto, este estudo tem por objetivo caracterizar o perfil do desempenho das habilidades de coordenação motora dos escolares com transtorno autístico.

\section{Método}

Este trabalho foi aprovado pelo Comitê de Ética em Pesquisa da Faculdade de Filosofia e Ciências da Universidade Estadual Paulista - FFCl UNESP/Marília - SP, sob o protocolo de número 0262/2010.

Participaram deste estudo 6 escolares com diagnóstico de transtorno autístico, avaliados previamente por equipe interdisciplinar (neurologia, neuropsicologia, fonoaudiologia e terapia ocupacional), que apresentam prejuízos no desempenho acadêmico, social e de lazer, atendidos semanalmente no 
Centro de Estudos da Educação e Saúde - CEES/FFC/UNESP-Marília - SP no Estágio Supervisionado Curricular de Distúrbios da Linguagem, na faixa etária de 5 anos e 5 meses a 10 anos e 9 meses de idade, matriculados regularmente em escolas públicas municipais da cidade de Marília-SP. mentos:

Para a realização deste estudo foram utilizados os seguintes procedi-

a) Termo de Consentimento: Conforme resolução do Conselho Nacional de Saúde CNS 196/96, anteriormente ao início das avaliações, os pais ou responsáveis dos pacientes selecionados assinaram o termo de Consentimento Pós-Informado para autorização da realização do estudo.

b) Escala de Desenvolvimento Motor (EDM - ROSA NETO): O procedimento aplicado compreende um conjunto de provas muito diversificadas e de dificuldades graduadas, conduzindo a uma exploração minuciosa de diferentes habilidades motoras do desenvolvimento da criança. Entre elas destaca-se coordenação motora em motricidade fina (MF), motricidade global (MG), equilíbrio $(E)$, esquema corporal (EC), organização espacial (OE), organização temporal (OT) e Lateralidade (LA).

Os testes foram realizados segundo os critérios descritos no manual do próprio autor (ROSA NETO, 2002), por uma terapeuta ocupacional, em uma sala de atendimento do Centro de Estudos da Educação e Saúde - CEES/FFC/ UNESP-Marília em uma única sessão com duração média de trinta minutos.

Os resultados foram analisados seguindo os critérios definidos na EDM (ROSA NETO, 2002), ou seja, por meio do cálculo do quociente motor geral (QMG). Esse cálculo foi realizado por meio da divisão da idade motora geral (IMG) pela idade cronológica (IC) e multiplicado por cem (QMG= IMG/IC x 100), resultando em uma pontuação referente à função motora geral. Com a obtenção desta pontuação, esta foi classificada de acordo com parâmetros estabelecidos na tabela 1. 
Tabela 1 - Classificação da Escala de Desenvolvimento Motor - EDM (ROSA NETO, 2002).

\begin{tabular}{c|c}
\hline Pontuação & Classificação \\
\hline 130 ou mais & Muito Superior \\
\hline $120-129$ & Superior \\
\hline $110-119$ & Normal Alto \\
\hline $90-109$ & Normal Médio \\
\hline $80-89$ & Normal Baixo \\
\hline $70-79$ & Inferior \\
\hline 69 ou menos & Muito Inferior \\
\hline
\end{tabular}

Depois do término deste estudo, todos os pais ou responsáveis pelos escolares foram informados sobre os resultados por meio de relatórios e orientados sobre a necessidade do desenvolvimento de atividades com as funções motoras avaliadas tanto em casa como em sala de aula.

\section{Resultados}

Os resultados na tabela 2 apresentam a comparação entre a idade cronológica e a idade motora geral, o quociente motor geral e a classificação do desenvolvimento motor dos escolares segundo a Escala do Desenvolvimento Motor (ROSA NETO, 2002).

Na comparação entre a idade cronológica (IC) e a idade motora geral (IMG), pode-se observar diferença entre elas, de forma que, a idade motora geral está muito abaixo da idade cronológica, com variação entre elas de 8 meses (sujeito III) a 3 anos (sujeito I) de diferença.

Em relação ao quociente motor geral (QMG) e a classificação do desenvolvimento motor, pode-se observar que $3(50 \%)$ escolares apresentaram desenvolvimento motor muito inferior ao esperado para a idade cronológica, 1 $(16,6 \%)$ escolar apresentou desenvolvimento motor inferior ao esperado para a idade cronológica, $1(16,6 \%)$ escolar apresentou o desenvolvimento motor normal baixo para a idade cronológica e $1(16,6 \%)$ escolar apresentou o desenvolvimento motor normal médio para a idade. 
Paola Matiko Okuda - Andrea Regina Nunes Misquiatti - Simone Aparecida Capellini

Tabela 2: Distribuição da Idade cronológica (IC), idade motora geral, quociente motor geral e classificação do desenvolvimento motor dos escolares.

\begin{tabular}{c|c|c|c|c}
\hline Sujeitos & IC & IMG & QMG & Classificação do D. M. \\
\hline I & $5 a 9 m$ & $3 a 2 m$ & 55 & Muito inferior \\
\hline II & $10 a 9 m$ & $7 a 9 m$ & 72 & Inferior \\
\hline III & $9 a 9 m$ & $8 a 11 m$ & 91 & Normal Médio \\
\hline IV & $6 a 5 m$ & $4 a$ & 62,3 & Muito inferior \\
\hline V & $6 a 1 m$ & $5 a$ & 82,19 & Normal Baixo \\
\hline VI & $5 a 5 m$ & $3 a 2 m$ & 58,46 & Muito Inferior \\
\hline
\end{tabular}

Legenda: IC: idade cronológica; IMG: idade motora geral; QMG: quociente motor geral; Clas. do D. M.: classificação do desenvolvimento.

Os resultados da tabela 3 apresentam a comparação entre a idade cronológica e a idade motora por função dos escolares com transtorno autístico, e a comparação entre as idades das funções motoras de cada escolar com transtorno autístico.

Os resultados mostraram que todos os escolares apresentaram as idades motoras fina (MF), global (MG), equilíbrio (Eq), esquema corporal/rapidez $(E C / r)$, organização espacial (OE) e organização temporal/linguagem (OT/l) abaixo da idade cronológica (IC).

As funções motoras de cada escolar mostram que um mesmo escolar pode apresentar idades motoras diferentes em cada uma das funções avaliadas. Essas diferenças podem variar em meses ou anos, como observado nos sujeitos I, II, III, V e VI; ou não apresentar variação como o sujeito IV (MF = MG = Eq $=\mathrm{EC} / \mathrm{r}=\mathrm{OE}=\mathrm{OT} / \mathrm{l})$. 
Tabela 3: Distribuição dos resultados referentes à Idade Motora por função avaliada

\begin{tabular}{c|c|c|c|c|c|c|c}
\hline Sujeitos & IC & MF & MG & Eq & EC/r & OE & ОT/I \\
\hline I & $5 a 9 m$ & $3 a$ & $3 a$ & $3 a$ & $4 a$ & $3 a$ & $3 a$ \\
\hline II & $10 a 9 m$ & $8 a$ & $8 a$ & $9 a 6 m$ & $5 a$ & $8 a$ & $8 a$ \\
\hline III & $9 a 9 m$ & $9 a 6 m$ & $8 a 6 m$ & $9 a 6 m$ & $6 a$ & $10 a$ & $10 a$ \\
\hline V & $6 a 5 m$ & $4 a$ & $4 a$ & $4 a$ & $4 a$ & $4 a$ & $4 a$ \\
\hline$V$ & $6 a 1 m$ & $4 a$ & $5 a 6 m$ & $5 a 6 m$ & $5 a$ & $5 a$ & $5 a$ \\
\hline$V I$ & $5 a 5 m$ & $3 a 6 m$ & $3 a$ & $3 a 6 m$ & $3 a$ & $3 a$ & $3 a$ \\
\hline
\end{tabular}

Legenda: IC: idade cronológica; MF: motor fino; MG: motor global; Eq: equilíbrio; EC/r: esquema corporal/rapidez; OE: organização espacial; OT/l: organização temporal/linguagem.

\section{Discussão}

Com base nos dados obtidos, observou-se que os escolares com transtorno autístico deste estudo apresentam idade motora geral abaixo do esperado para a idade cronológica, corroborando os estudos na literatura nacional (FERNANDES, 2008; WOFF, 2008; LIMA et al., 2001) e internacional (CORREIA, 2006; DEWEY; CANTELL; CROWFORD, 2007; LARSON et al., 2008; WILMUT, 2009; GABBARD; CAÇOLA, 2010; KOPP; BECKUNG; GILLBERG, 2010), que apontam que o atraso no desenvolvimento motor é uma característica comórbida do transtorno autístico.

Como se pôde observar nos achados deste estudo, os escolares apresentaram discrepância entre as idades cronológicas e as idades motoras fina (MF) e global (MG), o que pode estar relacionado com as alterações em diversas disfunções motoras, como: alterações nos movimentos, como os movimentos realizados na deambulação (andar nas pontas dos pés), alterações nos reflexos, movimentos associados, como mexer o corpo para a frente e para traz quando executa uma atividade, atraso nas aquisições motoras, má coordenação e desajeitamento geral, também leva a dificuldades significantes nas tarefas de vida diária, no lazer e nas tarefas escolares, incluindo dificuldades com a escrita, além dos jogos imitativos e jogos sociais, necessários para o desenvolvimento dos comportamentos sociais, comunicação e aprendizagem de habilidades de vida diária (CAPELLINI; SOUZA, 2008; FERNANDES, 2008; WOLFF, 2008; MAGALLÓN; NARBON, 2009; FUENTES et al., 2010).

É nos primeiros anos escolares que as noções de esquema corporal e organização espacial independente do corpo se estruturam, permitindo que a criança adquira a capacidade de compreender as relações entre as noções 
espaciais (direita, esquerda, frente e atrás) externas e referentes ao próprio corpo (MACEDO; ANDREUCCI; MONTELLI, 2004). Segundo Lima (2001), problemas no desenvolvimento do equilíbrio, esquema corporal e motricidade global fazem com que as crianças não planejem antecipadamente seus atos motores e, dessa forma, apresentam dificuldades com a orientação corporal e localização. Também por esses motivos, segundo Dewey (2007), escolares com transtorno autístico apresentam dificuldades em imitação de gestos, atividade que contribui para a aquisição e internalização de movimento corretos.

As atividades motoras estão diretamente relacionadas ao funcionamento de estruturas cerebrais, como os lobos frontais e temporais, o cerebelo, a amígdala, o hipocampo, o corpo caloso e o giro do cíngulo (LEVIT et al., 2003; ZILBOVÍCIUS; MERESSE; BODDAERT, 2006; MELO et al. 2010), estruturas estas envolvidas no ato motor voluntário, como mastigar, andar, escrever, e movimento involuntário, como piscar e salivar. Recentemente, estudos de neuroimagem em crianças com transtorno autístico, revelaram diminuição da ativação dos neurônios espelho na área de Broca, que poderiam estar envolvidos na representação do significado de movimentos, bem como no córtex parietal direito, o que pode contribuir para a codificação dos aspectos sinestésicos do movimento, o que, consequentemente, contribui para o atraso no desenvolvimento motor desses escolares (DEWEY, 2006).

Segundo Gabbard (2009), escolares com TDC, como os escolares com transtorno autístico, apresentam alteração na captação, assimilação e representação da imagem motora interna, também conhecida como imagem sinestésica, que é um processo cognitivo ativo durante o qual a representação de uma determinada ação é reproduzida internamente na memória de trabalho sem qualquer saída motora, em outras palavras, é um ensaio interno dos movimentos sem qualquer movimento físico evidente, conjuntamente com um mapeamento visual do ato motor, com isso, esses escolares acabam por apresentar reduzida capacidade de realizar ações voluntárias com movimentos muito elaborados partindo de sua própria imagem motora, dificultando, assim, suas experiências e estimulações externas.

As experiências motoras da criança são decisivas na elaboração progressiva das estruturas que aos poucos dão origem às formas supe-riores de

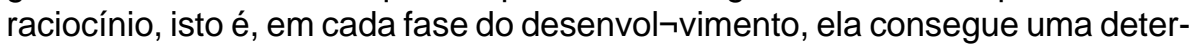
minada organização mental que lhe permite lidar com o ambiente. Pode-se assim afirmar que, em termos de evolução, a motricidade é uma condição de adaptação importante. A falta de experiências pode retardar e limitar a capacidade perceptiva do indivíduo (FERNANDES, 2008; LARSON et al.,2008; GABBARD; CAÇOLA, 2010).

Para Lucena et al. (2010), há uma necessidade de introduzir no cotidiano escolar programas de estimulação motora específicos que envolvam a lateralidade funcional e a organização espacial das crianças como meio de prevenir e intervir em prováveis distorções no processo de aprendizagem desde os 
primeiros anos de vida, isto porque o contexto escolar torna-se mais importante do que a influência da classe social familiar no ajustamento educacional e psicossocial na infância (SACKER; SCHOON; BARTLEY, 2002).

Com base nessa descrição e na necessidade de proporcionar experiências motoras aos escolares com transtorno autístico, a literatura nacional (TAMANAHA, 2006; MONTEIRO; PICK; VALENTINI, 2008) e internacional (FUENTES; MOSTOFSKY; BASTIAN, 2009; KOPP; BECKUNG; GILLBERG, 2010; GABBARD; CAÇOLA, 2010) refere que para proporcionar a internalização dos movimentos, ou seja, a organização e sequenciação do ato motor, a utilização de atividades motoras, percepto-viso-motoras e sensório-motoras, atividades lúdicas organizadas e estruturadas, jogos simbólicos, jogos grupais, atividades sinestésicas, atividades que explorem o desenvolvimento de esquema corporal, equilíbrio, organização espacial, organização temporal e coordenação motora fina, atividades para atenção seletiva e atividades que utilizem a criatividade são as mais eficazes e eficientes quando utilizadas como estratégia de tratamento, possibilitando, assim, a utilização funcional (organizada e para um fim determinado) da ação motora, pois fornecem uma base sólida dos movimentos e fundamental para que o escolar com transtorno autístico adquira conhecimento sobre o que seu corpo pode fazer e como fazê-lo, base necessária para a realização das atividades de vida diária, social, escolar e lúdica.

Dessa forma, assim como coloca Fernandes (2008), é através da aquisição de novos meios de expressão, conscientização e possibilitando o acesso a funções importantes para a comunicação e socialização que o escolar com transtorno autístico passará a explorar seus ambientes adquirindo maiores experiências, ou seja, é através do desenvolvimento do movimento como parte da criança com transtorno autístico que permitirá que ela se comunique com o mundo e também se organize enquanto sujeito para dar conta da sua participação na sociedade.

\section{Conclusão}

Com base nos achados deste estudo, devemos considerar a hipótese de que os escolares com transtorno autístico apresentam quadro de Transtorno do Desenvolvimento da Coordenação (TDC) em comorbidade, uma vez que os mesmos apresentam dificuldades em atividades que exijam destreza, agilidade e coordenação decorrentes do TDC e não do quadro de autismo. Isto pode ser uma das explicações para o prejuízo no desempenho acadêmico, social e no lazer desses escolares.

Assim, faz-se necessário que profissionais da área da educação, terapeutas ocupacionais e fisioterapeutas enfoquem as necessidades motoras, viso-motoras e psicomotoras desses escolares em situação de aprendizagem, visando proporcionar a diminuição do impacto das manifestações comportamentais e sociais e, dessa forma, favorecer melhor qualidade de vida social e acadêmica, além de maior independência funcional de pessoas com transtorno autístico. 
Paola Matiko Okuda - Andrea Regina Nunes Misquiatti - Simone Aparecida Capellini

\section{Referências}

Associação Americana de Psiquiatria (APA). Manual diagnóstico estatístico de transtornos mentais. (DSM-IV-TR). Poro Alegre: Artes Médias; 2002.

BARNHART, R. C. et al. Developmental coordination disorder. Physicatric Therapy, v. 83, p. 722-731, 2003.

CAPELLINI, A. S.; SOUZA, A. V. Avaliação da função motora fina, sensorial e perceptiva em escolares com dislexia. In: Sennye AL, Capovilla FC, Montiel, JM. Transtornos da aprendizagem da avaliação à reabilitação. São Paulo: Artes Médicas, 2008, p. 55-64.

CORREIA, N. M. M. Estudo exploratório dos níveis de coordenação motora em indivíduos com perturbações do espectro autístico. [Dissertação de Mestrado em Ciências do Desporto - Área em especialização em atividade física adaptada] Universidade do Porto - Faculdade de Desporto, 2006.

DEWEY, D.; CAMTELL, M.; CROWFORD, S. Motor and gestural performance in children with autism spectrum disorders, developmental coordination disorder and/or attention deficit hyperactivity disorder. Journal of the International Neuropsychological Society, 13:246-256, 2007.

DIAMOND, A. Close interrelation of motor development and cognitive development and of the cerebellum and prefrontal cortex. Children Development, 71:44-56, 2000.

FERNANDES, F. S. O corpo no autismo. PSIC - Revista de Psicologia da Vetor Editora, v. 9, n. 1, p. 109-114, Jan./Jun. 2008.

FUENTES, C. T.; MOSTOFSKY, S. H.; BASTIAN, A. J. Children with autism show specific handwriting impairments. Neurology, 73:1532-7, 2009.

GABBARD, C. Studying action representation in children via motor imagery. Brain and Cognition, 71:234-239, 2009.

GABBARD, C.; CAÇOLA, P. Los niños con trastorno del desarrollo de la coordinación tienen dificultad on la representación de las acciones. Revista de Neurologia, 50: 33-8, 2010.

GETCHELL, N. et al. Comparing children with and without dyslexia on the movement assessment battery for children and the test of gross motor development. Percepition and Motor Skills, 105: 207-214, 2007.

KOLB, B.; WHISHAW, I. Q. Neurociências do comportamento. Barueri: Editora Manole; 2002.

KOPP, S.; BECKUNG, E.; GILLBERG, C. Developmental coordination disorder and other motor control problems in girls with autism spectrum disorder and/or attention-deficit/hyperactivity disorder. Research in Developmental Disabilities, 31:350-361, 2010. 
LARSON, J. C. G. et al. Acquisition of internal models of motor tasks in children with autism. Brain, November; 131(Pt 11): 2894-2903, 2008.

LEVITT, J. G. et al. Cortical sulcal maps in autism. Cerebral Cortex, 13(7):728$35,2003$.

LIMA, C. B. et al. Equilíbrio dinâmico: influência das restrições ambientais. Revista Brasileira de Cineantropometria \& Desempenho Humano. v. 3, n. 1, p. 83-94, 2001.

LUCENA, N. M. G. et al. Lateralidade maual, ocular e dos membros inferiores e sua relação com déficit de organização espacial em escolares. Estudos de Psicologia, Campinas, v. 27, n. 1, p. 3-11, jan./mar. 2010.

MACEDO, C. S.; ANDREUCCI, L. C. MONTELLI, T. C. B. Alterações cognitivas em escolares de classe sócio-econômica desfavorecida: resultados de intervenção psicopedagógica. Arquivos de Neuro-psiquiatria. 2004, 62 (1): $852-$ 57.

MAGALHÃES, L. C.; NASCIMENTO, V. C. S.; REZENDE, M. B. Avaliação da coordenação e destreza motora-ACOORDEM: etapas de criação e perspectivas de validação. Revista Brasileira de Terapia Ocupacional. 15: 17-25, 2004.

MAGALLÓN S.; NARBONA J. Detección y estudios específicos en el trastorno de aprendizaje procesal. Revista de Neurolgía. v. 48 (Supl 2): S71-S76, 2009.

MELO, T. M. et al. Relações entre alterações anatomofuncionais do sistema nervoso central e autismo. Revista CEFAC, São Paulo, v. 8, n.1 , 36-41, jan./ mar., 2006.

MONTEIRO, T. R.; Pick, R. K.; Valentini, N. C. Responsabilidade social e pessoal de crianças participantes de um programa de intervenção motora inclusiva. Temas sobre Desenvolvimento, 16(94):202-14, 2008.

Organização mundial da saúde (OMS). Classificação de transtornos mentais e de comportamento do CID-10: descrições clínicas e diretrizes diagnósticas. Porto Alegre: Artes Médicas, 1993.

ROSA NETO, F. Manual de avaliação motora. Porto Alegre: Artemed; 2002.

SACKER, A.; SCHOON, I.; BARTLEY,M. Social inequality in educational achievement and psychosocial adjustment throughout childhood: magnitude and mechanisms. Social Science and Medicine. 55 (5): 863-80, 2002.

CHIRMER, C. R.; FONTOURA, D. R.; NUNES, M. L. Distúrbios da aquisição da linguagem e da aprendizagem. Jornal de Pediatria. Porto Alegre, v. 80, n. 2, abr. 2004.

STEINLIN, M. Cerebellar disorders in childhood: cognitive problems. Cerebellum, 7(4):607-10, 2008. 
TAMANAHA, A. C. et al. A atividade lúdica no autismo. Distúrbios da Comunicação, São Paulo, 18(3): 307-312, dez., 2006.

TSENG, M. H. et al. Cooccurrence of problems in activity level, attention, psychosocial adjustment, reading and writing in children with developmental coordination disorder. International Journal of Rehabitation Research, 30(4):327-32, 2007.

VIHOLAINEN, $\mathrm{H}$. et al. Development of early motor skills and language in children at risk of familial dyslexia. Developmental Medicine Child of Neurology, 44: 761-769, 2002.

WILMUT, K. Selection and assessment of children with developmental coordination disorder. Developmental Medicine \& Child Neurology, DOI: 10.1111/j.1469-8749.2009.03347.x. Published. Acesso em: jun. 2009.

WOLFF, A. C. Avaliação da maturação percepto-cognitiva e do comportamento motor em crianças com transtorno autista: indicações ao trabalho do educador. Revista electrónica de investigación y docencia (REID), 1, set. p. 71-98, 2008.

ZILBOVICIUS, M.; MERESSE, I.; BODDAERT, N. Autismo: neuroimagem. Revista Brasileira de Psiquiatria. v. 28, supl.1, São Paulo: maio 2006.

Correspondência

Paola Matiko Martins Okuda - Rua Aviador Gomes Ribeiro, 23-50, apto. 26, Jardim Brasil. CEP 17011-067. Bauru, São Paulo, Brasil.

E-mail: paolaokuda@yahoo.com.br

Recebido 18 de junho de 2010

Aprovado em 21 de outubro de 2010 